\title{
"Strange Kinetics" in the Temperature Dependence of Methionine Ligand Rebinding Dynamics in Cytochrome c
}

\author{
Ping Zhang, Steven Wooseok Ahn, and John E. Straub* \\ Department of Chemistry, Boston University, Boston, Massachusetts 02215, United States
}

\begin{abstract}
The temperature dependence of methionine ligand dissociation and rebinding dynamics in cytochrome $\mathrm{c}$ in aqueous solution has been studied using classical molecular dynamics simulation. Results are compared with previous study of rebinding dynamics at $300 \mathrm{~K}$ in water in order to understand how the change of protein environment and the underlying protein energy landscape influence the dynamics. Rebinding dynamics at 77,180 , and $300 \mathrm{~K}$ exhibits changes in both time scale and mechanism as the protein and solvent undergo a dynamic "glass transition". At each temperature, the rebinding dynamics yields a subset of trajectories that undergo fast rebinding as well as a subset of trajectories that undergo slower rebinding. At $300 \mathrm{~K}$ in water, both a fast ( $4.0 \mathrm{ps})$ and slow (14.6 ps) rebinding is observed. While fast rebinding occurs from a "downward" (heme pointing) substate of the methionine, the slow rebinding involves interconversion between an "upward" substate, from which rebinding cannot occur, and the downward substate. At lower temperatures $(77$ and $180 \mathrm{~K})$, the upward dissociated substate was not observed due to the high barrier imposed by the "frozen" protein structure. However, a slow rebinding phase is observed at both 77 and $180 \mathrm{~K}$ and is associated with a process of trapping in downward but "binding forbidden" substates with subsequent slow dynamical conversion to "binding competent" substates from which rebinding is relatively rapid. Distinctive rebinding dynamics at 77 and $180 \mathrm{~K}$ suggest that different rebinding time scales are predetermined by the protein and solvent structural arrangement prior to photodissociation, which causes either fast rebinding (about 2 ps) or slow ( $>50$ ps) rebinding. Suggestions for future experiments to further probe the role of dynamic heterogeneity in the kinetics of methionine ligand binding in cytochrome $\mathrm{c}$ protein are proposed.
\end{abstract}

\section{INTRODUCTION}

Most studies of the temperature and viscosity dependence of ligand rebinding dynamics in heme proteins have focused on bimolecular rebinding of diatomic ligands to heme proteins. ${ }^{1-29}$ Those studies have provided an understanding of the rich kinetics observed in diatomic ligand dissociation and binding in terms of the diversity of underlying protein conformational substates. ${ }^{7,8,30-35}$ The seminal work of Agmon, ${ }^{8,30,31}$ Frauenfelder, $^{32,33}$ Champion, ${ }^{36,37}$ and their co-workers focused on understanding nonexponential binding kinetics and the role played by the diverse ensemble of protein conformations at varying temperatures. The transition from inhomogeneous to homogeneous kinetics observed experimentally has been explained through a number of theoretical models. Champion and co-workers ${ }^{34,35}$ studied temperature-dependent heme kinetics by measuring the $\mathrm{CO}$ and $\mathrm{NO}$ rebinding dynamics and concluded that the nonexponential kinetic response of myoglobin is not necessarily due to protein conformational substates, emphasizing the role of the local heme dynamics.

The kinetics of photodissociation of the methionine ligand to the heme of cytochrome $c$ have received less attention. Methionine (Met80) ligand in cytochrome c (cyt c, see Figure 1) is covalently linked to the protein backbone, making the unimolecular rebinding dynamics quite different from bimo- lecular diatomic ligand rebinding. ${ }^{38}$ Initial excitation of the heme leads to dissociation of the methionine ligand on a quintet excited-state surface followed by non-adiabatic dynamics (possibly involving intermediate triplet states), leading to rebinding of the ligand to the heme on the ground singlet state. Studies of diatomic ligand rebinding, such as nanosecond time scale rebinding of $\mathrm{CO}$ in myglobin, suggest that at room temperature the interconversion of protein conformations is much faster than the rebinding time scales, resulting in a homogeneous environment that promotes exponential rebinding dynamics. Early experimental studies at room temperature on the photodissociation of Met80 in reduced $\mathrm{Fe}$ (II) cyt $\mathrm{c}$ were carried out by Champion and coworkers $^{39}$ where rebinding of Met80 was observed to occur on a time scale of $6.2 \mathrm{ps}$. The same system was studied by Kruglik and co-workers, ${ }^{40}$ and both fast and slow rebinding, with time constants of 4.8 and 16 ps, were observed. A later work by Vos and co-workers ${ }^{41}$ suggested a 5-7 ps heme-residue bond formation time scale in a variety of six-coordinate heme proteins. In recent work, Zhong and co-workers ${ }^{42}$ observed

Received: January 15, 2013

Revised: April 24, 2013

Published: April 30, 2013 

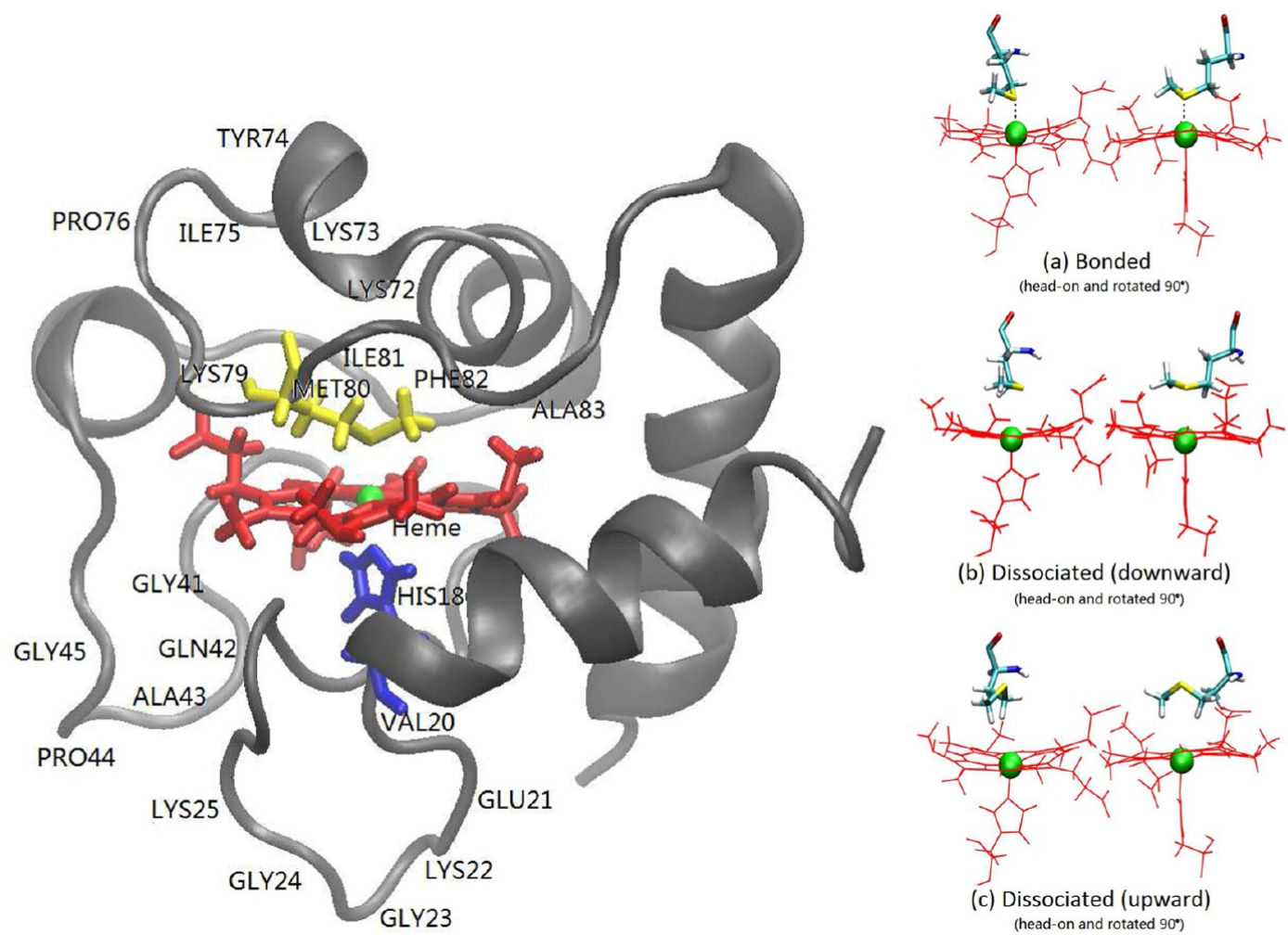

(a) Bonded (head-on and rotated $90^{\circ}$ )

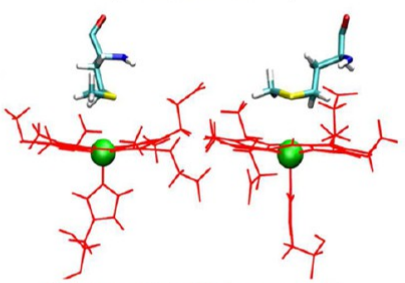

(b) Dissociated (downward) (head-on and rotated $90^{\circ}$ )

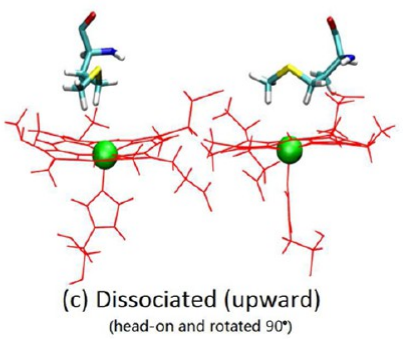

Figure 1. Left: cytochrome $\mathrm{c}$ and the heme pocket reaction center. Right: conformational substates essential to the reaction kinetics are shown for the reaction center (heme, Met80, and His18) as (a) bonded, and dissociated (b) downward and (c) upward conformations.

Met80 ligand rebinding with a time constant of approximately 7 ps. Subsequently, our theoretical study ${ }^{38}$ demonstrated that Met80 rebinding in cytochrome $c$ displays a more complex kinetics in which the reaction dynamics include interconversion of the Met80 ligand between upward (rebinding forbidden) and downward (rebinding competent) substates following photodissociation. Interconversion between these nonreactive (upward) and reactive (downward) substates represents, at first glance, a kind of local inherent kinetics of the ligand, weakly coupled to the global protein conformational change.

This study of protein reaction dynamics is designed to explore the conjectures that (1) localized ligand dynamics play a major role in the observed ligand rebinding kinetics at room temperature while (2) more delocalized global conformational transitions play a critical role in rebinding dynamics when the temperature is low. We present the study of Met80 rebinding dynamics in water at various temperatures $(77,180$, and $300 \mathrm{~K})$ in order to identify the molecular dynamics that underlies rebinding dynamics and the observed nonexponential population decay at low and high temperatures.

At high temperature, it has been proposed that the observed double exponential decay is due to the interconversion of the Met80 residue between rebinding forbidden and rebinding competent substates. At low temperatures, the nonexponential decay is primarily caused by an inhomogeneous distribution of protein (as opposed to Met80 ligand) conformations, leading to a distribution of rebinding time scales but not the interconversion between Met80 upward and downward substates observed at room temperature. Dynamics analysis allows for the identification of key protein coordinates that are correlated to the Met 80 substate interconversion, providing an explanation for why the rebinding forbidden upward substate of the Met80 ligand is present at $300 \mathrm{~K}$ in water but not observed at lower temperature. The study also demonstrates how the dynamics of the reaction center (the atoms defining the local heme pocket) and more global motions contribute to the overall rebinding dynamics.

\section{METHODS}

2.1. Molecular Dynamics Simulation of Ligand Dissociation and Rebinding. The nonadiabatic dynamics model and the underlying potential energy for the bound (singlet) and dissociated (quintet) surfaces have been described elsewhere. ${ }^{38}$ The sequence of 1HRC cytochrome $c$ from the PDB was used to define the human cytochrome c system, and the crystal structure was taken as the initial configuration. A cubic water box with 10440 TIP3P water molecules ${ }^{43}$ was added to surround the protein. The Stormer-Verlet ${ }^{44}$ algorithm and periodic boundary conditions were used in the CHARMM simulation program using the CHARMM28 force field. ${ }^{45}$ The particle-mesh Ewald method was used to calculate the electrostatic energy. All bonds with hydrogen atoms were constrained using SHAKE. ${ }^{46}$ The simulation time step was 1 fs.

A set of structures of the singlet state system (with sixcoordinate heme) was randomly chosen from an equilibrium trajectory at $300 \mathrm{~K}$ (as described above), and rapidly cooled to 180 and $77 \mathrm{~K}$. With the rapid cooling approach, a series of protein initial configurations sampling various local potential energy minima was preserved at low temperatures. To allow the system to relax after cooling, it is not possible to establish rigorous thermodynamic equilibrium sampling at the lower temperatures. Constant pressure and temperature (CPT) dynamics was used to establish a "local equilibration" starting from each structure. The final coordinates were collected at the end of each local equilibration run and taken to be the coordinates for initiation of the dynamic trajectories. The 
modified TIP3P water model was used at all temperatures. From each of these system configurations, a number of separate dissociation and rebinding trajectories were initiated, each having a unique set of initial velocities randomly sampled from the Maxwell distribution. A total of 500 trajectories for each condition was collected for analysis.

2.2. Analysis of Correlated Structural Fluctuations. Principal component analysis ${ }^{47}$ (PCA) is a method that effectively captures the correlation of motions in a system by eliminating uncorrelated noise. It was employed in our study to capture the correlation between the protein backbone and the Met80 motions resulting from photodissociation and rebinding.

To capture the Met80 flipping motions that define interconversion between upward (rebinding forbidden) and downward states at $300 \mathrm{~K}$, and transition between downward (rebinding competent) and bonded states, structures used for PCA were prepared in two ways:

(1) To represent the first $100-200$ fs of a dynamics trajectory following photodissociation at $300 \mathrm{~K}$, a series of structures was taken starting from the singlet equilibrium and including a set of intermediate structures representing the quintet dissociated configuration. A snapshot was taken every $10 \mathrm{fs}$, and roughly 20 frames were saved from each trajectory. A total of 2000 frames were analyzed using PCA. Figure 2 shows the concatenated trajectory in terms of the $\mathrm{Fe}-\mathrm{S}$ distance as a function of time.

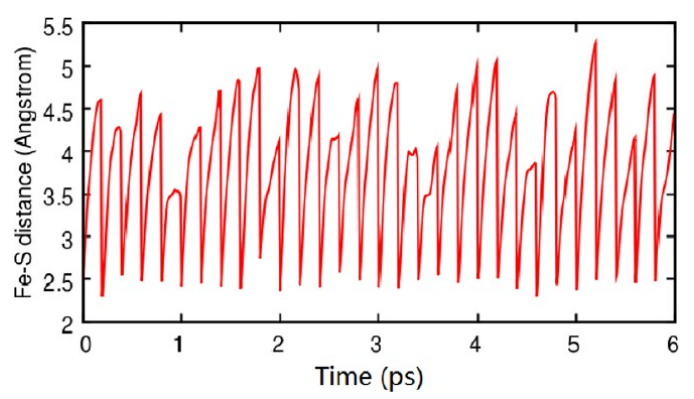

Figure 2. Concatenated trajectory formed from the initial $200 \mathrm{fs}$ of multiple dissociative reaction dynamics trajectories.

(2) To represent the reactant and product states, 3-4 ns equilibration trajectories in the singlet and quintet states were combined to create a hybrid trajectory, representing the equilibrium ensembles of bonded and dissociated states for PCA. This procedure was carried out for the protein in water at 300, 180, and $77 \mathrm{~K}$, respectively. Figure 3 shows a fragment of the singlet and quintet equilibration trajectories in terms of the

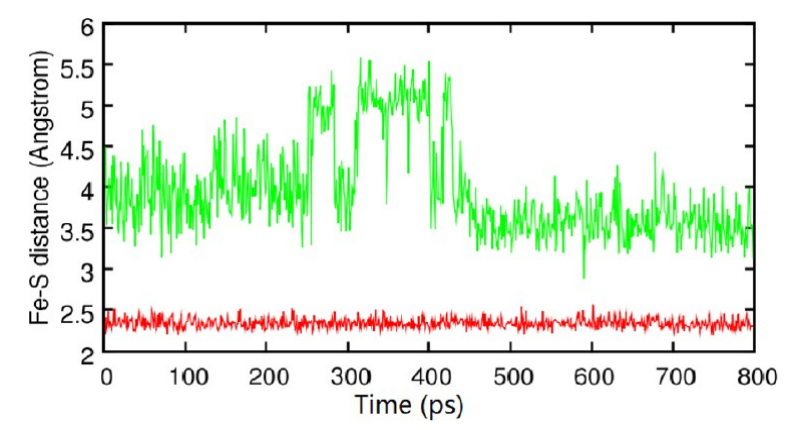

Figure 3. Fragment of singlet (red) and quintet (green) state equilibration trajectories at $300 \mathrm{~K}$ used for PCA.
$\mathrm{Fe}-\mathrm{S}$ distance as a function of time, indicating the involvement of the bonded, the dissociated downward, and the dissociated upward substates with regard to the Met80 motion in the hybrid trajectory. The quintet state trajectory shows interconversion between the upward and downward substates corresponding to large and small $\mathrm{Fe}-\mathrm{S}$ separation. The PCA over the hybrid trajectory captures key degrees of freedom in the Met80 motion in transitions between bonded and dissociated states.

Eigenvectors of the first principal component under each condition $(300,180$, and $77 \mathrm{~K})$ were used to construct a $4 \times 4$ dot-product matrix, to demonstrate the similarities and differences between each pair of vectors (see Table 2).

2.3. Analysis of Activation Energy Barriers. To capture the most essential degrees of freedom required for a transition from one state or substate to another, conjugate peak refinement $(\mathrm{CPR})^{48}$ was employed to calculate the reaction path from the reactant state - in this case, the downward (rebinding-competent) substate or the upward (rebinding forbidden) substate to the product state (in this case, the bonded state or the downward substate). This method requires the minimized reactant and product state structures, from which a reaction path is calculated in terms of a set of geometries. The energy and structural changes along the path can then be analyzed. Structures in the reactant and product states were selected from actual dynamics trajectories, and were each minimized to the nearest local potential energy minimum.

In the standard Arrhenius reaction rate theory, ${ }^{8,49-51}$ the reaction rate $k$ is proportional to the Boltzmann probability $\exp \left(-E / k_{\mathrm{B}} T\right)$, where $E$ is the activation energy, $k_{\mathrm{B}}$ is the Boltzmann constant, and $T$ is the temperature. Dynamics in a "glassy" system may be associated with a distribution of energy barriers, leading to kinetics that display a non-Arrhenius temperature dependence.

\section{RESULTS}

\subsection{Time Evolution of Photodissociation and Rebind-} ing. The $\mathrm{Fe}-\mathrm{S}$ distance $R_{\mathrm{Fe}-\mathrm{S}}$ was monitored as the key reaction coordinate and indicator of photodissociation and rebinding. Typical rebinding trajectories at 77 and $180 \mathrm{~K}$ are shown in Figure 4. Trajectories attributed to $77 \mathrm{~K}$ are delineated with " $77 \mathrm{~K}$ ", and all others are attributed to 180 K. Each of the trajectories starts from a six-coordinated equilibrium structure. $R_{\mathrm{Fe}-\mathrm{S}}$ increases from the equilibrium bond length, $R_{\mathrm{Fe}-\mathrm{S}}=2.3 \AA$, to the dissociated distance, $R_{\mathrm{Fe}-\mathrm{S}}>$ $3.5 \AA$, within $100 \mathrm{fs}$ following photodissociation. Upon rebinding, $R_{\mathrm{Fe}-\mathrm{S}}$ rapidly returns to $2.3 \AA$ after fluctuating around 3.5-4 $\AA$ for a short period of time. Figure 4 also reveals that, following photodissociation at very low temperature, some of the trajectories reach a large $\mathrm{Fe}-\mathrm{S}$ separation (around $5 \AA$ ) due to a collision-like excitation. This large $\mathrm{Fe}-\mathrm{S}$ separation, however, does not survive long and quickly returns to shorter lengths. This indicates that at low temperature the surrounding protein environment does not undergo a structural rearrangement upon photodissociation that allows for the population of the Met80 upward (rebinding forbidden) substate. In fact, as discussed later, the activation energy for the transition from the downward to upward substate of the Met80 becomes much higher as the temperature is lowered and the protein and solvent undergo a dynamic glass-like transition.

Figure 4 also shows the survival probability of dissociated Met80 as a function of time, $S(t)$, exhibiting nonexponential 


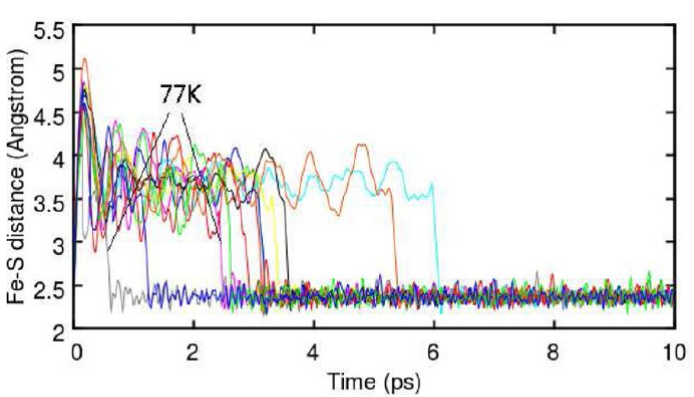

(a)

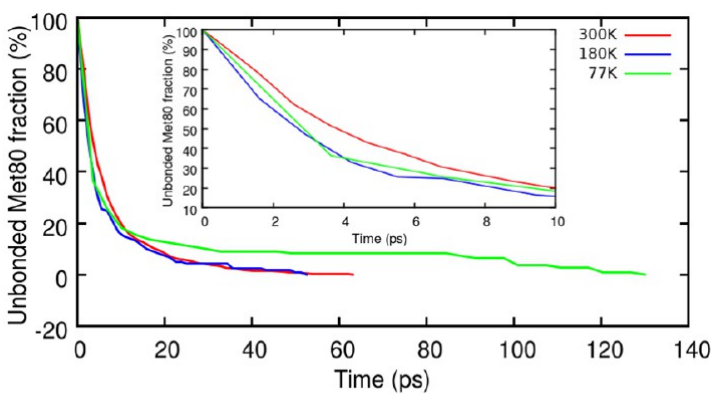

(b)

Figure 4. (a) Rebinding trajectories in water solvent at 180 and $77 \mathrm{~K}$ and (b) survival probability of dissociated Met 80 as a function of time for 300 , 180 , and $77 \mathrm{~K}$ in water.

decay at all temperatures. We have fitted the survival probability to a biexponential form

$$
S(t)=A_{1} \mathrm{e}^{-\left(t / t_{1}\right)}+A_{2} \mathrm{e}^{-\left(t / t_{2}\right)}
$$

a power-law function

$$
S(t)=\frac{1}{\left(1+\frac{t}{t_{0}}\right)^{n}}
$$

and a stretched exponential decay

$$
S(t)=\mathrm{e}^{-(t / \tau)^{\beta}}
$$

The parameters for the fits to our data are presented in Table 1, and the plots are shown in Figure 6. At each temperature, the $\chi^{2}$ values are best for the biexponential fit, nearly as good for the power-law fit, and less good for the stretched exponential form.

Table 1. Parameters for Best Fits to Survival Probability Data at Different Temperatures Using Biexponential, PowerLaw, and Stretched Exponential Functions

$\begin{array}{lcccccccc} & \tau & \beta & t_{0} & n & A_{1} & t_{1} & A_{2} & t_{2} \\ 300 \mathrm{~K} & 6.0 & 0.81 & 15.5 & 3.1 & 73.0 & 4.2 & 27.0 & 15.0 \\ 180 \mathrm{~K} & 4.2 & 0.64 & 5.0 & 1.7 & 72.0 & 2.6 & 28.0 & 15.3 \\ 77 \mathrm{~K} & 2.2 & 0.30 & 0.83 & 0.62 & 83.2 & 2.8 & 16.8 & 77.7\end{array}$

The distributions of rebinding times observed at 180 and 77 $\mathrm{K}$ are shown in Figure 5. Over $80 \%$ of the trajectories at $77 \mathrm{~K}$ yield very fast rebinding, while the remaining fraction undergoes substantially slow rebinding (longer than 55 ps). Rebinding times longer than 55 ps were not observed at $180 \mathrm{~K}$. The semilog and log-log plots of rebinding survival probability at each temperature (see Figure 7) demonstrate highly heterogeneous kinetics at $77 \mathrm{~K}$. Due to a relatively greater time scale difference between the fast and slow rebinding, the $77 \mathrm{~K}$ plots in both Figure $7 \mathrm{a}$ and $\mathrm{b}$ reveal a flatter trend compared to those at 180 and $300 \mathrm{~K}$. The abrupt drop-off at 77 $\mathrm{K}$ in Figure $7 \mathrm{~b}$ near $\ln (t)=4.5$ corresponds to an "opening" of a channel for rebinding that contributes to the overall profile of the rebinding survival probability. It is clear that this onset of later delayed rebinding corresponds to a slow mode that is tied to fluctuations of the displaced methionine sulfur atoms relative to the heme iron. In this sense, a helpful analogy may be with dynamic facilitation in the relaxation of glasses, where relaxation in one local region of configuration space (relative displacement of methionine due to protein strain at lower temperature) facilitates the relaxation of another local region (sulfur-iron rebinding). ${ }^{52,53}$ The similarity of the plots at 180 and $300 \mathrm{~K}$ results from a coincidence of time scales as the rebinding kinetics at each temperature follows a distinctly different mechanism (discussed below).

The time scale distribution demonstrates the two characteristic changes in the distribution of rebinding times as temperature is lowered. (1) At lower temperatures, a larger fraction of trajectories rebinds rapidly and does so on a time scale that decreases as the temperature is lowered. (2) At lower temperatures, the time scale for rebinding of the slowest rebinding trajectories becomes longer as the temperature is lowered. Such nonexponential ligand rebinding kinetics has been said to result from protein conformational heterogeneity, created by quenching the sample from high temperature to low temperature. ${ }^{32,54}$ The rebinding time decay has been fitted using biexponential, power-law, and stretched exponential functions. The values of the fitting parameters at different temperatures are summarized in Table 1.

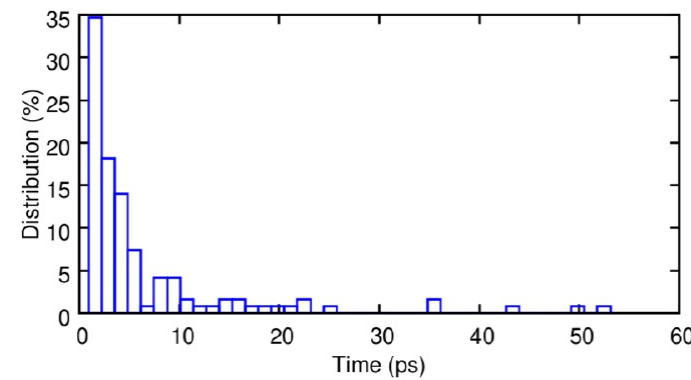

(a)

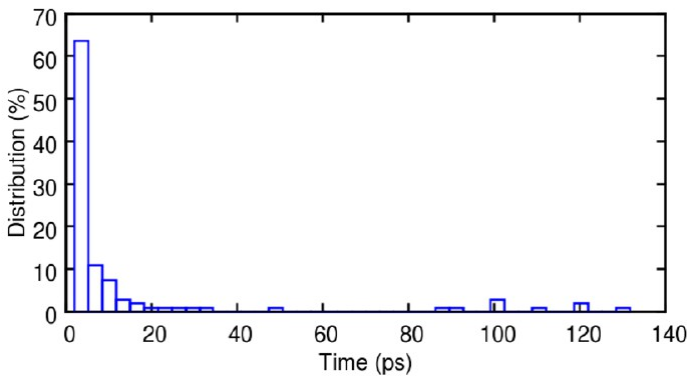

(b)

Figure 5. (a) Rebinding time distribution at $180 \mathrm{~K}$. (b) Rebinding time distribution at $77 \mathrm{~K}$, indicating fast, medium, and slow rebinding events. 

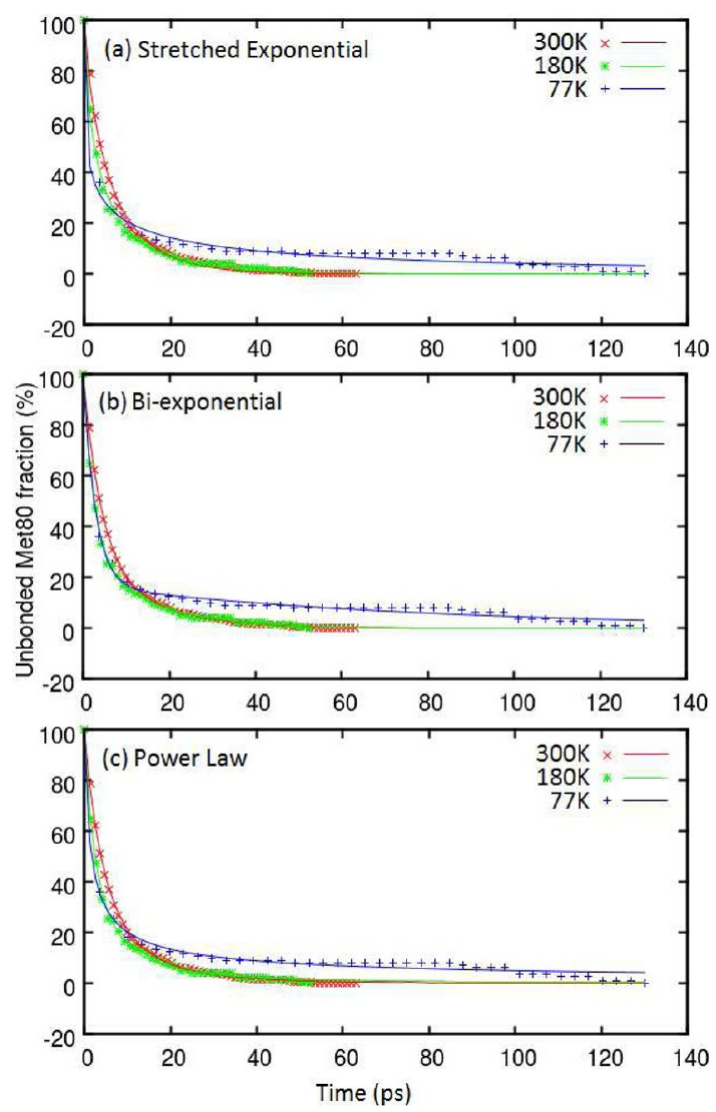

Figure 6. Unbonded Met80 survival probability as a function of time: data and fittings using parameters in Table 1.

Data for the stretched exponential function mostly clearly demonstrate a strong transition to more "glassy" dynamics at lower temperatures. Moreover, the stretched exponential fits to the survival probability show that (1) $\tau$ becomes smaller as the temperature is lowered (the slow rebinding trajectories rebind more slowly and the fastest rebinding trajectories rebind faster) and (2) $\beta$ becomes lower as the temperature is lowered (the system becomes increasingly heterogeneous in its dynamics). The $\beta=0.8<1$ at $300 \mathrm{~K}$ apparently results from the contribution of two rebinding channels to the overall mechanism of ligand rebinding. Similar trends are observed for the fit to the power-law function, where $t_{0}$ is observed to increase with temperature (like $\tau$ ) while $n$ also increases with temperature (as does $\beta$ ).
A previous study suggests that the time spent for interconversion between the upward and downward substates at $300 \mathrm{~K}$ in water leads to a slower rebinding time scale. The population of the upward substate was, however, not observed at 77 or $180 \mathrm{~K}$ in water solvated cyt c. Using the reaction coordinates, $R_{\mathrm{Fe}-\mathrm{S}}$ and $\theta$ defined in our previous study, examples of 2-D trajectories at each temperature are plotted in Figure 8. At 180 and $77 \mathrm{~K}$, only the downward substate is populated.

The dynamics of $R_{\mathrm{Fe}-\mathrm{S}}$ for the quintet state at various temperatures, in the absence of rebinding, is represented in Figure 9 in terms of the distributions of $R_{\mathrm{Fe}-\mathrm{S}}$. Compared to $300 \mathrm{~K}$ in water, $R_{\mathrm{Fe}-\mathrm{S}}$ at lower temperatures tends to explore the lower side of the downward substate where the centers of the peaks are near $3.5 \AA$. There is no distribution near $5 \AA$, which corresponds to the upward substate observed in watersolvated cytochrome $\mathrm{c}$ at $300 \mathrm{~K}$. It is reasonable to interpret the much faster rebinding component observed at low temperatures as a reflection of the frozen protein environment. The slow rebinding component observed at low temperatures cannot be explained using the mechanism identified for dynamics at $300 \mathrm{~K}$, due to the rigidity of the protein and inaccessibility of the Met80 upward substate. Our analysis suggests that the nonexponential decay in rebinding kinetics at both 180 and $77 \mathrm{~K}$ is largely determined by the protein conformation at the time of photodissociation.

3.2. Why Fast Rebinding at Lower Temperature Is Faster: Met80 Motions and Correlated Protein Motions. In contrast to bimolecular diatomic ligand rebinding, the dissociated Met80 ligand in cytochrome $\mathrm{c}$ is covalently linked to the protein backbone, making the reaction unimolecular. Following photodissociation, the Met80 ligand is trapped in the heme pocket surrounded by the heme and other residues (Figure 1). At $300 \mathrm{~K}$, the nonexponential rebinding kinetics are attributed to fluctuations between reactive and nonreactive conformational substates of the Met80 ligand. At lower temperature in water, the nonreactive substates are not visited with significant probability. This raises the question, why does the fast rebinding become more rapid as the temperature is lowered? To answer this question, it is helpful to explore the correlated motions of the protein upon photodissociation and rebinding using principal component analysis.

By combining the singlet and quintet equilibration trajectories at $300 \mathrm{~K}$, we were able to capture motions that are strongly correlated to the Met80 flipping motion in the first three principal components (see Methods). The motion of the transition or interconversion of Met80 among the bonded state,

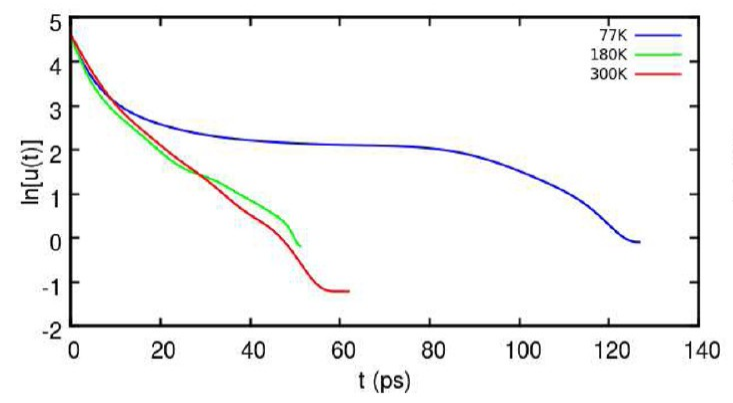

(a)

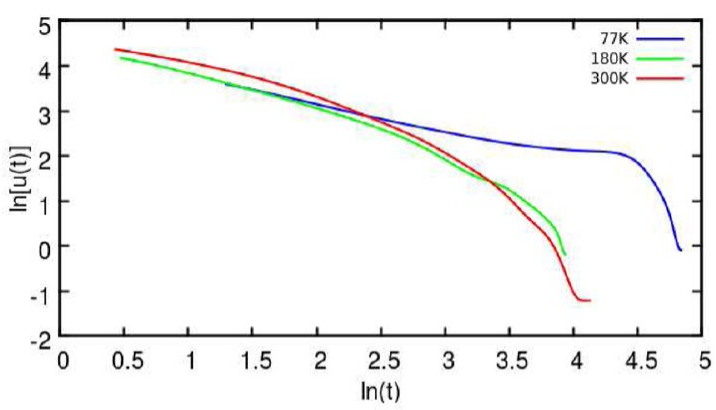

(b)

Figure 7. Rebinding survival probability of dissociated Met80 ligand as a function of time presented as (a) semilog plot (smoothed) and (b) log-log plot (smoothed). 

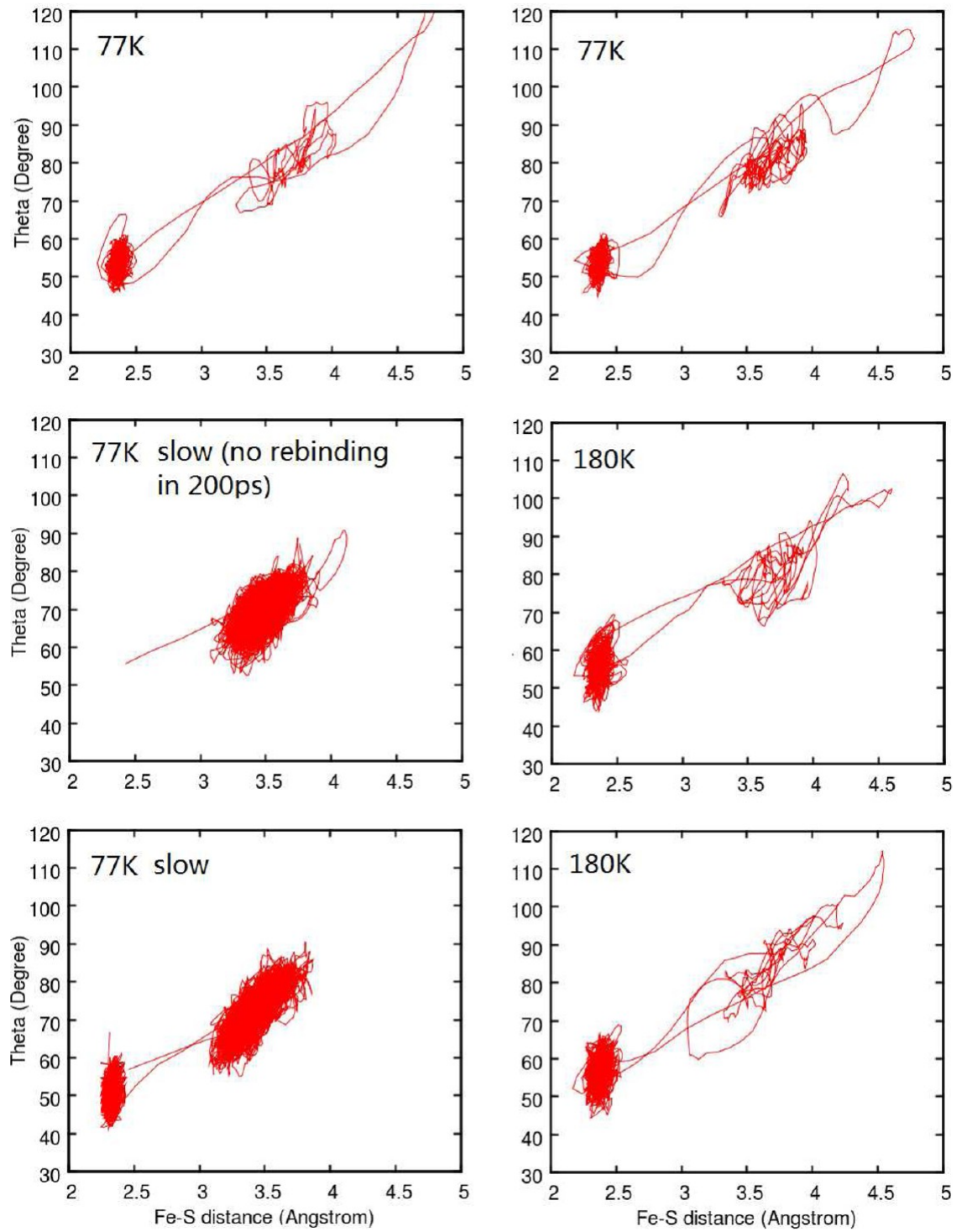

Figure 8. Two-dimensional projections of trajectories of Met80 rebinding ( $\theta$ in degrees, $R$ in $\AA$ ). The $\theta$ angle tracks the transition between downward $\left(\theta \approx 50^{\circ}\right)$ and upward $\left(\theta \approx 90^{\circ}\right)$ substates.

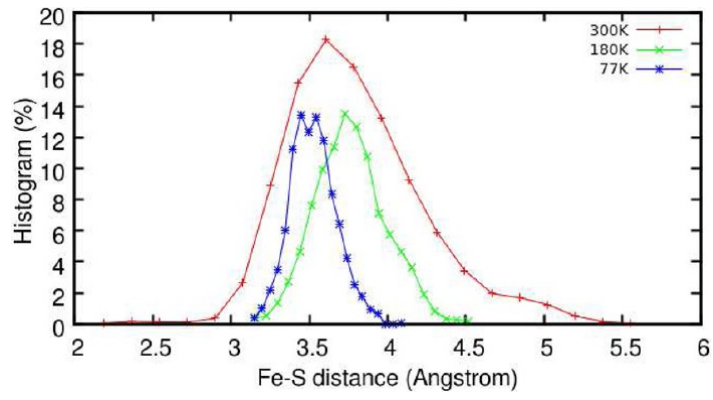

Figure 9. $R_{\mathrm{Fe}-\mathrm{S}}$ probability distributions for the protein in the dissociated quintet state only (no transitions to the singlet state for rebinding were allowed). The distributions show the downward substate population in the absence of the upward substate (with $\mathrm{Fe}-\mathrm{S}$ $=5 \AA$ ) for 77,180 , and $300 \mathrm{~K}$ in water.

the downward substate, and the upward substate can be understood in terms of three principal motions. (1) Separation of the center of mass of Met 80 and the heme, concurrent with the heme doming and the iron atom out-of-plane motion. (2)
The sulfur atom flipping between the bonded state and the downward reactive substate. During this process, the structure of Met80 does not change. (3) The S-C-C-C torsional motion, that reflects the interconversion between downward (reactive) and upward (nonreactive) substates, an interconversion between methionine rotamers.

Eigenvectors calculated from PCA describing motion in the protein backbone correlated to Met 80 motion are shown in Figure 10. Red dots follow the motion of heavy atoms in the protein backbone, showing the extent of motion of each heavy atom. The eigenvectors of the first three principal components of water solvated cyt $\mathrm{c}$ at $300 \mathrm{~K}$ show correlated motions in residue clusters $20-25,41-45,72-76$, and 79-83 (see Figure 1).

The eigenvectors of the first principal component for water solvated cyt $\mathrm{c}$ at 77 and $180 \mathrm{~K}$, derived from combining the singlet and quintet equilibration trajectories in each condition, do not show significant motions of the protein backbone correlated to the Met80 motion (see Figure 10). The protein environment is more frozen, slaved by the transition to "glassy" dynamics of the solvent, and restricted in motion. In particular, 


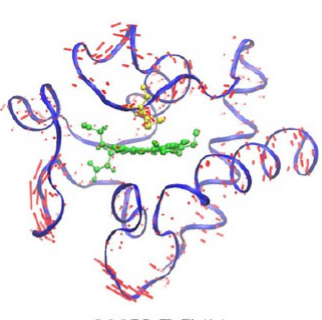

(a)

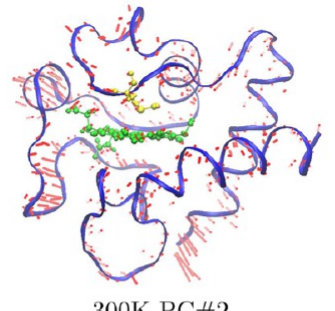

(b)

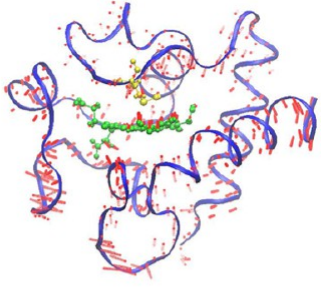

300K PC\#3

(c)

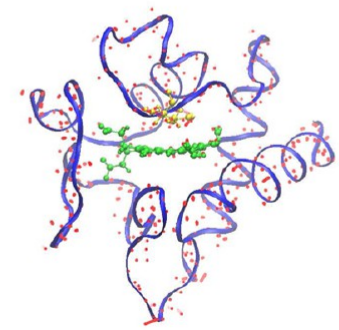

180K PC\#1

(d)

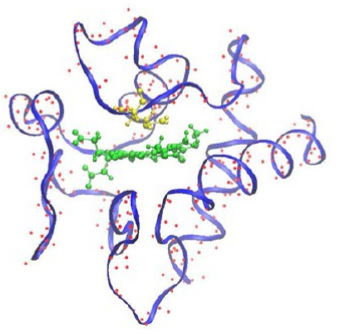

77K PC\#1

(e)

Figure 10. Illustrations of protein backbone motion correlated to the Met80 motion during structural transitions. Red dots indicate the displacement of each heavy atom in the backbone during rebinding. The first three eigenvectors calculated from PCA in the water solvated cytochrome $\mathrm{c}$ at $300 \mathrm{~K}$ (see part a, b, and c) may be compared with the first eigenvector calculated from PCA in the water solvated cytochrome $\mathrm{c}$ at (d) $180 \mathrm{~K}$ and (e) $77 \mathrm{~K}$.

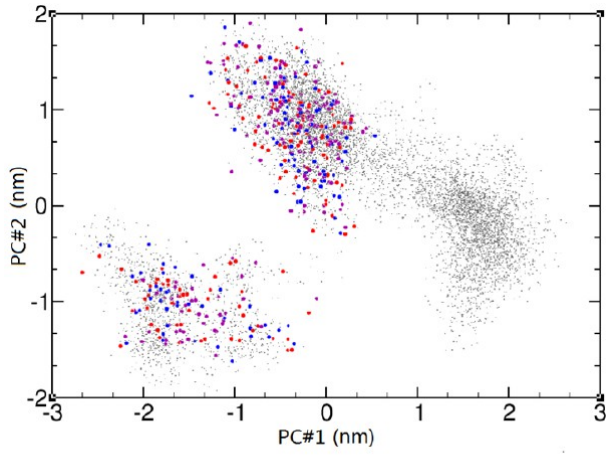

(a)

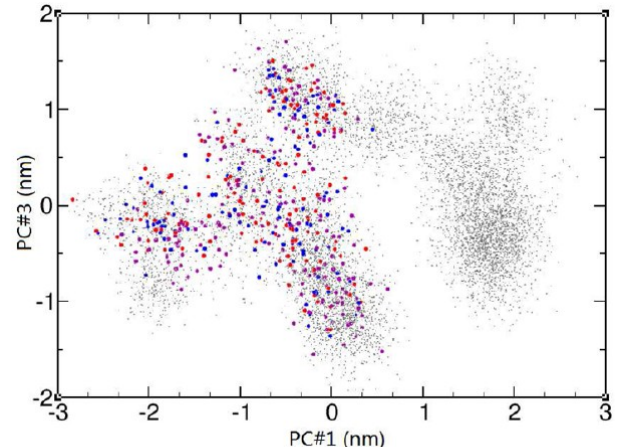

(b)

Figure 11. Gray dots: Projections of PCA trajectories (at $300 \mathrm{~K}$, in water) on principal component eigenvectors (a) PC\#1 and PC\#2 and (b) PC \#1 and PC \#3. Purple dots: projection of all initial structures used for rebinding dynamics runs at $300 \mathrm{~K}$ in water. Blue dots: projection of all quenched initial structures at $300 \mathrm{~K}$ cooled to $77 \mathrm{~K}$. Red dots: projection of all quenched initial structures at $300 \mathrm{~K}$ and cooled to $180 \mathrm{~K}$.

fluctuations in loop and helical regions, including those essential to rearrangement following dissociation to allow access to the upward ligand conformations, are suppressed. At these lower temperatures, large conformational rearrangements coupled to Met80 photodissociation and rebinding on the picosecond time scale are essentially eliminated.

The background gray dots in Figure 11 depict the projection of the water solvated cyt $\mathrm{c}$ trajectory at $300 \mathrm{~K}$ on the first and second (Figure 11a) and the first and third (Figure 11b) principal component eigenvectors. Two major clusters of dots in each of the plots demonstrate the separation of the bonded and dissociated configurations in each subspace.

Table 2 shows the dot product of each pair of first principal component eigenvectors derived from the analysis of the combined singlet and quintet state dynamics in the absence of rebinding. The eigenvector at $300 \mathrm{~K}$ has a negative dot product with those at lower temperatures. Eigenvectors at 180 and $77 \mathrm{~K}$ in water have positive dot products between each other. The negative dot product indicates that the correlated motion of the protein at $300 \mathrm{~K}$ in water is in the direction opposite to the others. This is a measure of the unique response from the
Table 2. Dot Product Matrix of the First Principal Component Eigenvector from Water Solvated Cytochrome c at 300,180 , and $77 \mathrm{~K}$

\begin{tabular}{lccc} 
& $300 \mathrm{~K}$ & $180 \mathrm{~K}$ & $77 \mathrm{~K}$ \\
$300 \mathrm{~K}$ & 1 & -0.17 & -0.1 \\
$180 \mathrm{~K}$ & -0.17 & 1 & 0.36 \\
$77 \mathrm{~K}$ & -0.1 & 0.36 & 1 \\
\hline
\end{tabular}

protein backbone to the Met80 motion undergoing transition between the downward (rebinding competent) and upward (rebinding forbidden) substates, motion that is not observed at 77 and $180 \mathrm{~K}$.

It is interesting to observe that the PCA of structures taken from the first 200 fs of each dynamics trajectory (Figure 2) does not capture any obvious correlation between motion of the Met80 and the protein backbone. This indicates that, during the fast nonequilibrium dynamics of photodissociation or rebinding, the rate of rebinding is predetermined by the protein structure at the time of dissociation. In the bonded state, the protein backbone is necessarily in a conformation 
consistent with ligand rebinding. Following photodissociation, protein structure rearrangement can take place ${ }^{55}$ when the temperature is high, allowing the protein to explore binding competent as well as binding forbidden substates. At low temperature where the conformational change of the protein and solvent behave more like a glass on the sub-ps time scale, the protein is essentially constrained to its neighboring potential energy minimum, exploring either binding competent substates or binding forbidden substates but not both. Binding competent substates lead to relatively fast rebinding, and binding forbidden substates lead to slow rebinding. Protein conformational sampling at $300 \mathrm{~K}$ becomes much faster, leading to an ambiguous boundary between the rebinding competent and rebinding forbidden substates. As a result, the fast rebinding is slowed and the slow rebinding occurs more rapidly due to enhanced access to the binding competent substates.

The distribution of initial structures used for rebinding dynamics runs at $300 \mathrm{~K}$, projected on the eigenvectors of the first three principal components (see purple dots in Figure 11), is restricted in the singlet region. In water solvent at $300 \mathrm{~K}$, picosecond time scale equilibration allows the protein to sample a diverse ensemble of bound conformations. For the study of rebinding dynamics at lower temperature, initial structures at $300 \mathrm{~K}$ in water were rapidly quenched to 77 and $180 \mathrm{~K}$ and allowed to equilibrate. The equilibrated structures are projected on the same three eigenvectors (blue and red dots in Figure 11). The overall distribution is similar to that at 300 $\mathrm{K}$, demonstrating the abundance of local energy minima in this quenched state distribution.

3.3. Minimum Energy Reaction Paths at 300 and $77 \mathrm{~K}$. In order to extract the most essential degrees of freedom involved in the transition in Met80 between upward and downward substates as well as the rebinding to the bonded state, we performed conjugate peak refinement (CPR) calculations to identify plausible transition paths. $\mathrm{CPR}$ requires minimum energy reactant and product structures. It is necessary to choose the reactant and product structures shortly before and after a transition, in order to preserve the minimized structures in the neighborhood of the representative conformational basins.

Structures in the upward substate, downward substate, and bonded state from four slow rebinding trajectories in water solvated cytochrome $\mathrm{c}$ at $300 \mathrm{~K}$ were chosen and minimized for $\mathrm{CPR}$ calculations. The reaction paths were projected on the $R-\theta$ two-dimensional surface obtained from our previous study of rebinding in water at $300 \mathrm{~K}$ (see Figure 12). The projections suggest that the $R$ and $\theta$ are two essential degrees of freedom that are informative in describing the transition from the dissociated state to the bonded state. Figure 12 indicates that, although the degrees of freedom from the solvent were eliminated in our CPR calculations, the key protein degrees of freedom along the reaction path are aligned with the two essential coordinates established from the minimum free energy path analysis presented in our study.

As the protein quickly samples various conformational basins at high temperature, the minimized reactant and product structures can fall into basins that are quite different in potential energy. This suggests that, at extremely low temperature, the energy barrier that the system must overcome to allow a transition from one substate to another can be large. In an actual dynamics run, the protein is typically in a transient high energy conformational state, allowing for transition from one

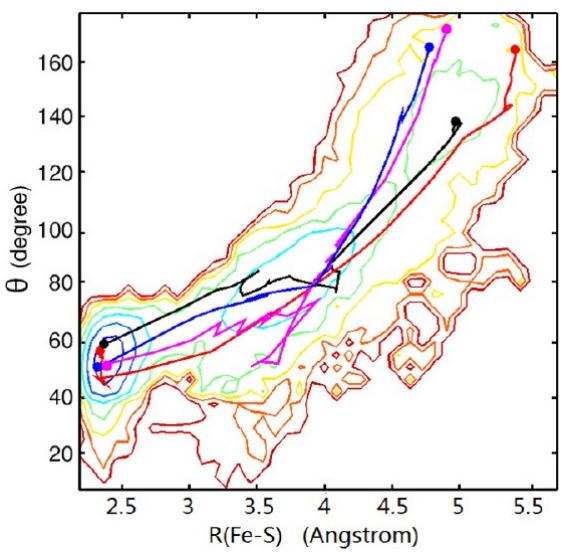

Figure 12. Projection of the minimum energy reaction paths of four rebinding trajectories in water solvated cytochrome $\mathrm{c}$ at $300 \mathrm{~K}$ on the $2 \mathrm{D}$ free energy map ( $\theta$ in degrees, $R$ in angstroms) obtained from our previous study. ${ }^{38}$

basin to another. Our previous analysis suggests that at room temperature the effective free energy barrier separating the upward (rebinding forbidden) and downward (rebinding competent) substates is only on the order of several $\mathrm{kcal} / \mathrm{mol}{ }^{38}$

3.4. Origins of Slow Rebinding at 77 and $180 \mathrm{~K}$. Apparently, for a trajectory that evolves in a conformational basin that impedes rebinding, the system must overcome a barrier to reach a basin that promotes rebinding. A major difference between rebinding-promoting basins and rebindingimpeding basins, in terms of the reaction barriers, can be interpreted from the generalized Landau-Zener formula introduced in our theoretical model. ${ }^{38}$ According to our energy criterion that defines the crossing zone, the energy difference between the singlet and quintet states, $\epsilon_{\text {diff }}$ must be less than 2 $\mathrm{kcal} / \mathrm{mol}$

During a dynamics run, the more frequently $\epsilon_{\text {diff }}$ reaches the $<2 \mathrm{kcal} / \mathrm{mol}$ range, the higher probability that the system will transition from one state to the other. Trajectories that display fast rebinding (within 2 ps) and slow rebinding (longer than 90 ps) at $77 \mathrm{~K}$ were collected separately. The energy difference between the singlet and quintet states, $\epsilon_{\text {diff, }}$ was calculated for configurations where Met 80 is dissociated, and the distributions are shown in Figure 13. The average $\epsilon_{\text {diff }}$ is $13.6 \mathrm{kcal} / \mathrm{mol}$ from those fast rebinding trajectories and $14.6 \mathrm{kcal} / \mathrm{mol}$ from slow

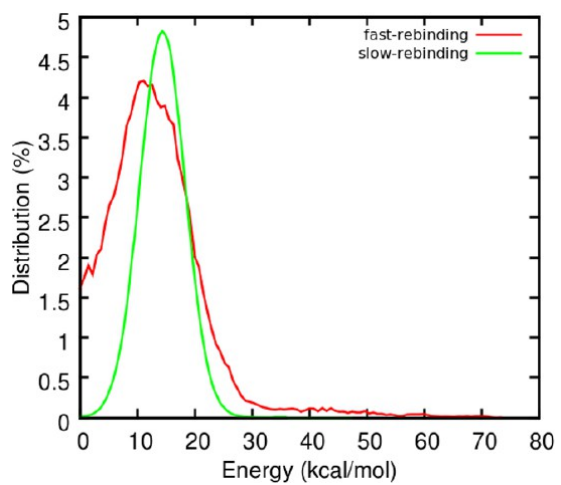

Figure 13. Distribution of $\epsilon_{\text {diff }}$ from a collection of fast and slow rebinding trajectories, repectively, at $77 \mathrm{~K}$ using quenched $300 \mathrm{~K}$ initial structures. The distribution of $\epsilon_{\text {diff }}$ that reaches the $<2 \mathrm{kcal} / \mathrm{mol}$ zone is $7 \%$ for fast rebinding trajectories and $0.15 \%$ for slow rebinding trajectories. 


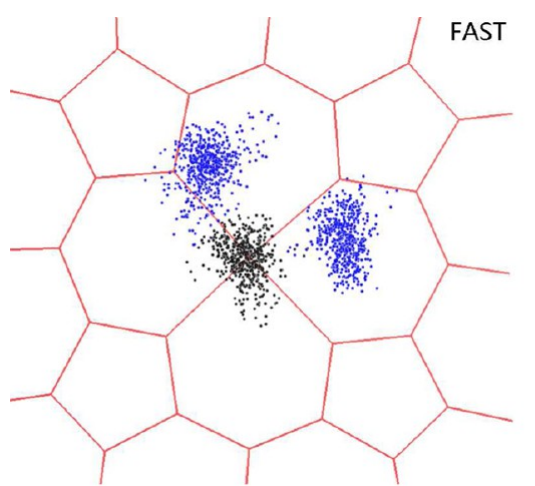

(a)

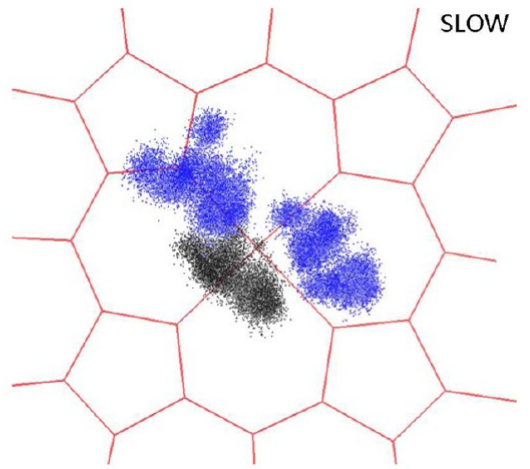

(b)

Figure 14. Distribution of positions of the sulfur atom (in black) and two carbon atoms (in blue) in the C-S-C group of Met80 from (a) a collection of fast rebinding trajectories at $77 \mathrm{~K}$ and (b) a collection of slow rebinding trajectories at $77 \mathrm{~K}$, all derived from quenched $300 \mathrm{~K}$ initial structures.

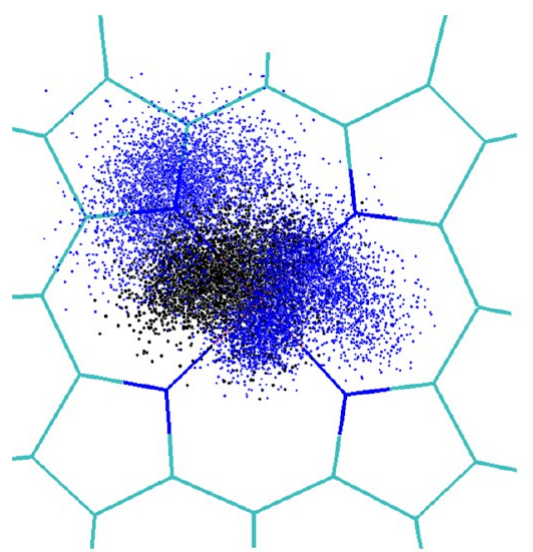

(a)

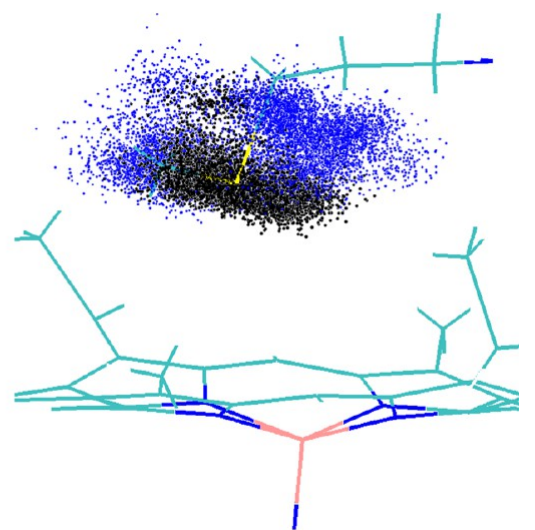

(b)

Figure 15. Distribution of positions of the sulfur atom (in black) and two carbon atoms (in blue) in the C-S-C group of Met80 derived from an equilibration trajectory in the quintet state (in the absence of rebinding) in water at $300 \mathrm{~K}$. Head on (upper) and rotated $90^{\circ}$ (lower).

rebinding trajectories. For fast rebinding, $\epsilon_{\text {diff }}$ that reaches the $<2 \mathrm{kcal} / \mathrm{mol}$ range comprises about $7 \%$ of the overall distribution. For slow rebinding, this number is only $0.15 \%$. Slow rebinding trajectories have fewer chances to reach the crossing zone before rebinding, compared with fast rebinding trajectories. What is responsible for the larger energy gap between the singlet and quintet states in the slow rebinding trajectories?

The essential degrees of freedom in the protein that contribute to various conformational basins are difficult to identify, especially when the motions are "collective", as in a backbone displacement or local fluctuation in free volume. However, since the degrees of freedom used to define the singlet and quintet states in our theoretical model are relatively few in number, ${ }^{38}$ it is possible to closely observe and identify how those degrees of freedom (used to define the singlet and quintet states) are influenced by the protein's global conformational motion and assess the impact on the reaction energy barrier (in this case, $\epsilon_{\text {diff }}$ ). Figure 14 illustrates the distribution of positions of the two carbon atoms (in blue) and the sulfur atoms (in black) in the $\mathrm{C}-\mathrm{S}-\mathrm{C}$ group of Met80 projected on the heme plane, the relative orientation of which is critical to ligand rebinding. For fast rebinding trajectories, the distribution of positions of the sulfur atom is more localized and aligned with the center of the heme (iron), whereas for slow rebinding trajectories the position distribution of the sulfur is shifted away from the center of the heme (iron). The overall distribution of all three atoms (C, S, and $\mathrm{C}$ ) in slow rebinding trajectories is more delocalized than that in fast rebinding trajectories. For rebinding to occur, the $\mathrm{C}-\mathrm{S}-\mathrm{C}$ group must reorient to allow the sulfur atom to align with the center of the heme. A more localized and better aligned (with iron) position of the sulfur promotes rapid rebinding. Similarly, a shifted and delocalized distribution of the positions of the sulfur atom makes the rebinding more difficult. The shift of the sulfur atom position distribution is a clear indication of the protein backbone structural shift away from configurations that promote fast rebinding. If the initial structure (quenched from $300 \mathrm{~K}$ ) is in a conformational basin in which the protein backbone is shifted, the Met80 residue will be displaced away from the center of the heme upon dissociation, resulting in slow rebinding.

Figure 15 shows the distributions of the positions of the C$\mathrm{S}-\mathrm{C}$ atoms from an equilibration trajectory in the quintet state (no rebinding allowed) at $300 \mathrm{~K}$ in water solvent. The overall distribution of each atom (C, S, and C) is more delocalized than the distributions at $77 \mathrm{~K}$ due to the involvement of the Met80 downward and upward conformational substates (absent in our simulations at lower temperatures). The distributions in Figure 14 are two subsets of the distributions in Figure 15. The overall distribution at $300 \mathrm{~K}$ (Figure 15) is more delocalized, indicating that there are lower barriers between configurations that promote or impede rebinding. In fact, the boundary 
between conformations becomes ambiguous. The fundamentally different nature of the dissociated configurations at high and low temperatures reflects the heterogeneity of ligand rebinding time scales at low temperature and the relative homogeneity observed at high temperature in water solvent.

The Met80 upward conformational substate was not observed at $180 \mathrm{~K}$, and the double-exponential decay observed in the rebinding kinetics at $180 \mathrm{~K}$ (see Figure 4) has an origin and mechanism that is fundamentally different from the doubleexponential decay observed at $300 \mathrm{~K}$ in water. The latter is due to the interconversion of the Met80 downward (rebinding competent) and upward (rebinding forbidden) substates. Comparison with rebinding at $77 \mathrm{~K}$ (see Figure 4) indicates that, as the temperature is raised (to $180 \mathrm{~K}$ ), the rebinding kinetics become more homogeneous and exponential-like. We observe that, with increasing temperature, the fast component of rebinding becomes slower, the slow component becomes faster, and the overall rebinding time scale becomes shorter. Given the slow rebinding time scale of 15.2 ps at $180 \mathrm{~K}$, one may expect the slow rebinding time scale at $300 \mathrm{~K}$ in water to be faster than 15.2 ps. However, in our previous study, slow rebinding observed at $300 \mathrm{~K}$ was on the time scale of $14.6 \mathrm{ps}$. This is essentially the same time scale observed at $180 \mathrm{~K}$ (15.2 ps) but with a fundamentally different origin.

To describe the structural difference in the heme doming at 300 and $77 \mathrm{~K}$, the $\mathrm{N}_{\mathrm{HEME}}-\mathrm{Fe}-\mathrm{N}_{\mathrm{His} 18}$ angle (see Figure 16) in

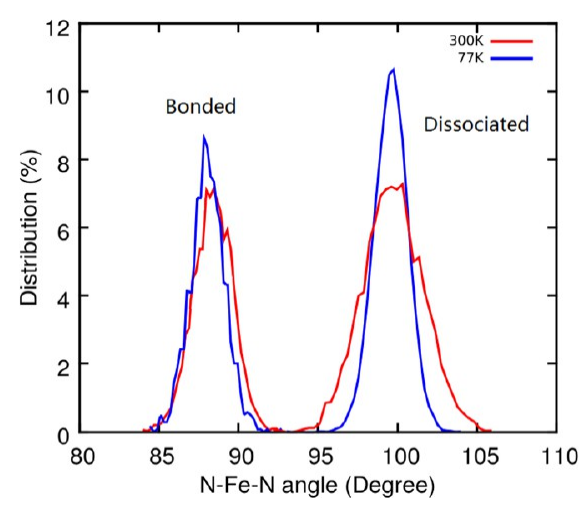

Figure 16. Distribution of $\mathrm{N}_{\mathrm{HEME}}-\mathrm{Fe}-\mathrm{N}_{\mathrm{His18}}$ angle in the bonded and dissociated states at $77 \mathrm{~K}$ (blue) and $300 \mathrm{~K}$ (red).

the bonded and dissociated states is used to indicate the displacement of the iron atom out of the heme plane (as a larger iron out-of-plane displacement is correlated with a greater $\mathrm{N}_{\mathrm{HEME}}-\mathrm{Fe}-\mathrm{N}_{\mathrm{His18}}$ angle for the range of structures explored in our simulated dynamics). The $\mathrm{N}-\mathrm{Fe}-\mathrm{N}$ angle displays a broader distribution (in the range $93-105^{\circ}$ ) in the dissociated state at $300 \mathrm{~K}$ than at $77 \mathrm{~K}$, although the center position of each peak is found to be near $99^{\circ}$. This broader distribution at $300 \mathrm{~K}$ may be attributed to a broader range of heme-pocket atom fluctuation in the dissociated state including the His 18 residue. While a role for proximal histidine tilting in discriminating ligand binding in hemoglobin has been established, ${ }^{56}$ our results suggest that the His18 tilting does not contribute in a similar way to explain different time scales at different temperatures in the event of the Met80 rebinding in cyt $c$, as the degree of the His18 tilting in the bonded and dissociated state at each temperature reveals no significant difference in our study. In the bonded state, the $\mathrm{N}-\mathrm{Fe}-\mathrm{N}$ angle distributions at 300 and $77 \mathrm{~K}$ are similar (in the range $85-93^{\circ}$ ) with the center of each peak near $88^{\circ}$. We have also compared the $\mathrm{N}-\mathrm{Fe}-\mathrm{N}$ distribution calculated from dissociated state configurations at $77 \mathrm{~K}$, taken from super fast and super slow trajectories, respectively. The result does not reveal a significant difference in the domed heme structure on the time scale of 100 ps. It appears that the structural fluctuation of the heme does not play a significant role in determining the broad distribution of rebinding time scales at low temperatures observed in our study. The degree of heme doming in terms of the iron atom out-of-plane displacement in cyt c $(0.35 \AA$ in our previous study $\left.{ }^{38}\right)$ is significantly less than that in myoglobin $\left(0.5 \AA^{11,17}\right)$, suggesting that less energy is required for iron displacement during rebinding. It is reasonable to believe that a combination of the more confined motion of the dissociated Met80 ligand and a relatively small iron out-of-plane displacement promotes relatively fast rebinding rates.

3.5. Activation Energy for Transition between Upward to Downward Substates. To further explore the role of the protein environment on the transition of the Met80 from the upward to downward substate, a number of Met80 upward configurations were randomly chosen from a quintet equilibration trajectory at $300 \mathrm{~K}$. Each configuration was quenched to lower temperatures and allowed to equilibrate. Figure 17 shows the distribution of $R_{\mathrm{Fe}-\mathrm{S}}$ during 60 ps of equilibration starting with upward configurations. Few transitions from the upward to downward substate are observed at $77 \mathrm{~K}$. The fraction of the upward substate increases as the temperature rises. As described earlier, all equilibration trajectories were run for the same duration. If we assume that the prefactor $A$ in the Arrhenius equation $A \exp \left(-E / k_{\mathrm{B}} \mathrm{T}\right)$ is

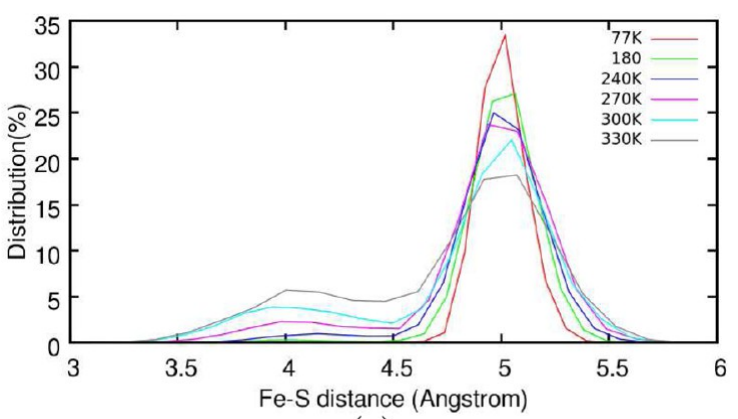

(a)

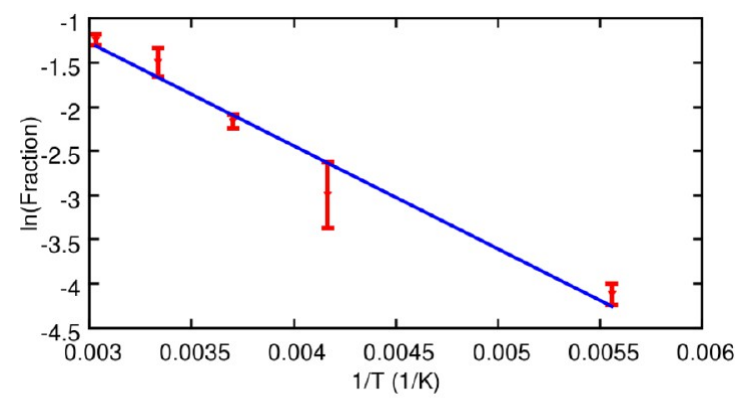

(b)

Figure 17. (a) Distribution of upward and downward substates in terms of $\mathrm{Fe}-\mathrm{S}$ distance at various temperatures. Equilibration started with upward configurations and was allowed to evolve for a fixed period of time at each temperature. The downward state population grows as the temperature increases. (b) The logarithm of the downward fraction is fitted as a linear function of the inverse temperature. 
insensitive to temperature change, the reaction rate constant $k$, that describes transition from the upward to the downward substate, is proportional to the fraction of the downward substate. The resulting estimate of the activation energy $E$ (see Figure 17 ) is $3.5 \mathrm{kcal} / \mathrm{mol}$.

The activation energy for the transition from the downward to upward substate is expected to be much higher. As suggested from the $R_{\mathrm{Fe}-S}$ distribution in our previous study, the fraction of trajectories accessing the upward substate transition at $300 \mathrm{~K}$ in water is roughly $10 \%,{ }^{38}$ while the fraction of the downward substate formed from the upward state (see Figure 17) is about $22 \%$. Similarly, as indicated in Figure 8, transition from the downward state to the upward state following photodissociation is not observed in water at 77 or $180 \mathrm{~K}$, while the fraction of the downward state formed from the upward state at 77 and $180 \mathrm{~K}$ are 0.2 and 5\%, respectively. This suggests that the transition barrier from the downward substate to the upward substate is too high for transitions to be observed on the picosecond time scale.

\section{DISCUSSION AND CONCLUSION}

Met80 rebinding in cytochrome $\mathrm{c}$ has been studied as a function of temperature $(300,180$, and $77 \mathrm{~K})$ using molecular dynamics simulations. The generalized Landau-Zener surfacehopping algorithm ${ }^{38}$ has been employed to perform reaction dynamics. Nonexponential ligand rebinding kinetics is observed at all temperatures, with a rebinding mechanism that is distinctly different at $300 \mathrm{~K}$ than at lower (180 and $77 \mathrm{~K}$ ) temperatures. The distinctive rebinding behavior at lower temperatures strongly suggests that rebinding time scales are predetermined by the details of the particular conformational substates of the protein at the time of photodissociation.

The protein conformation samples various basins in energy landscape of the singlet state at $300 \mathrm{~K}$. Some of the basins promote rebinding after photodissociation, whereas other basins impede rebinding. Upon photodissociation, the system is excited to the quintet state, but the global structures retain their original basins. When the temperature is very low, sampling becomes difficult. Some trajectories will rebind quickly as they remain in the promoting basins after photodissociation, which yields fast rebinding. In contrast, trajectories in the impeding basins yield slow rebinding, as prior to rebinding the system must undergo a transition to a promoting basin. When the temperature is increased, trajectories in the impeding basins may cross over barriers to reach promoting basins, and yield shorter time scales for the slowest rebinding trajectories. When the temperature is increased further, sampling becomes relatively fast, there are no distinct promoting or impeding basins, and rebinding becomes more homogeneous. At high enough temperatures, a second reaction channel, involving access to the "upward" substate of Met80, leads to "biexponential" kinetics.

Our analysis has identified essential steps in the process of rebinding at all temperatures. In the dissociated state, for rebinding to occur, the protein must access conformations with significant energy overlaps in the quintet and singlet states. At $300 \mathrm{~K}$, this may involve transition between upward and downward states of Met80 ligand. At 180 and $77 \mathrm{~K}$, this may involve transition from downward binding-forbidden substates to binding-allowed substates. In our study, the barriers between substates are caused by shifts of the protein backbone that displace Met80 from alignment above the heme center consistent with $\mathrm{Fe}-\mathrm{S}$ bond formation. The heme doming motion does not appear to play a significant role in contributing to the energy gap difference between fast and slow rebinding trajectories. Furthermore, the displacement of the iron from the heme plane in the dissociated state appears to be insensitive to temperature change.

In considering the nonadiabatic transition probability, once the energy criterion is satisfied, we recognize that the LandauZener transition probability is temperature dependent. Temperature dependence arises through the distribution of speeds in crossing the transition region as well as the temperature dependence of the spin-orbit coupling. We have compared the time derivative of the potential energy at low and high temperatures, finding only a small difference. The spin-orbit coupling in the Landau-Zener formula is temperature dependent, and a detailed study of the effect has been carried out by Gerstman. ${ }^{57}$ In our study, the spin-orbit coupling was taken to be constant across all temperatures.

As has been noted by Gruebele and cowokers, ${ }^{58}$ the exploration of "strange kinetics" in biomolecular dynamics is of fundamental importance. Bimolecular diatomic ligand rebinding kinetics in heme proteins has shown rich dynamical behavior. While we observe nonexponential kinetics for Met80 ligand rebinding in cytochrome $\mathrm{c}$ at all temperatures, the origin of nonexponential kinetics, including transitions in the mechanism of rebinding, appear to be distinct from those proposed for diatomic ligand rebinding in heme proteins. There are relatively few systems that have been studied over a wide range of temperature in a way that allows for a thorough exploration of the role of features of the energy landscape in determining nonexponential kinetics, with the richest systems involving ligand-protein rebinding. It will be of great interest to see the predictions of this study tested by experiment, and for experiment to build a body of data for the temperature dependence of ligand rebinding kinetics in this unique dynamical system.

\section{AUTHOR INFORMATION}

\section{Corresponding Author}

*E-mail: straub@bu.edu.

\section{Notes}

The authors declare no competing financial interest.

\section{ACKNOWLEDGMENTS}

We are grateful for the generous support of our research by the National Science Foundation (CHE-114676 and CHE0750309) and the Boston University Center for Computational Science. J.E.S. thanks Martin Gruebele and Markus Meuwly for helpful discussions.

\section{REFERENCES}

(1) Negrerie, M.; Kruglik, S. G.; Lambry, J. C.; Vos, M. H.; Martin, J. L.; Franzen, S. Role of Heme Iron Coordination and Protein Structure in the Dynamics and Geminate Rebinding of Nitric Oxide to the H93G Myoglobin Mutant: Implication for Nitric Oxide Sensors. J. Biol. Chem. 2006, 281, 10389-10398.

(2) Walther, M.; Raicu, V.; Ogilvie, J. P.; Phillips, R.; Kluger, R.; Miller, R. J. Determination of the Fe-CO Bond Energy in Myoglobin Using Heterodyne-Detected Transient Thermal Phase Grating Spectroscopy. J. Phys. Chem. B 2005, 109, 20605-20611.

(3) Silkstone, G.; Jasaitis, A.; Wilson, M. T.; Vos, M. H. Ligand Dynamics in an Electron Transfer Protein. Picosecond Geminate Recombination of Carbon Monoxide to Heme in Mutant Forms of Cytochrome c. J. Biol. Chem. 2007, 282, 1638-1649. 
(4) Mizutani, Y.; Kitagawa, T. Ultrafast Dynamics of Myoglobin Probed by Time-Resolved Resonance Raman Spectroscopy. Chem. Rec. 2001, 1, 258-275.

(5) McMahon, B. H.; Stojkovic, B. P.; Hay, P. J.; Martin, R. L.; Garcia, A. E. Microscopic Model of Carbon Monoxide Binding to Myoglobin. J. Chem. Phys. 2000, 113, 6831-6850.

(6) Margulis, C. J.; Guallar, V.; Sim, E.; Friesner, R. A.; Berne, B. J. A New Semiempirical Approach to Study Ground and Excited States of Metal Complexes in Biological Systems. J. Phys. Chem. B 2002, 106, $8038-8046$

(7) Yamashita, T.; Bouzhir-Sima, L.; Lambry, J. C.; Liebl, U.; Vos, M. H. Ligand Dynamics and Early Signaling Events in the Heme Domain of the Sensor Protein Dos from Escherichia coli. J. Biol. Chem. 2008, 283, 2344-2352.

(8) Agmon, N.; Hopfield, J. J. CO Binding to Heme Proteins: A Model for Barrier Height Distributions and Slow Conformational Changes. J. Chem. Phys. 1983, 79, 2042-2053.

(9) Nutt, D. R.; Karplus, M.; Meuwly, M. Potenial Energy Surfaces and Molecular Dynamics of MbNO: Existence of an Unsuspected FeON Minimum. J. Phys. Chem. B 2005, 109, 21118-21125.

(10) Berlina, Y. A.; Fischer, S. F.; Chekunaevb, N. I.; Goldanskii, V. I. Non-Exponential Non-Arrhenius Relaxation in the Course of CO Rebinding to Heme Proteins. Chem. Phys. 1995, 200, 369-385.

(11) Meuwly, M.; Becker, O. M.; Stote, R; Karplus, M. NO Rebinding to Myoglobin: A Reactive Molecular Dynamics Study. Biophys. Chem. 2002, 98, 183-207.

(12) Banushkina, P.; Meuwly, M. Free-Energy Barrier in MbCO Rebinding. J. Phys. Chem. B 2005, 109, 16911-16917.

(13) Nutt, D. R; Meuwly, M. Studying Reactive Processes with Classical Dynamics: Rebinding Dynamics in MbNO. Biophys. J. 2006, 90, 1191-1201.

(14) Straub, J. E.; Karplus, M. Molecular Dynamics Study of the Photodissociation of Carbon Monoxide from Myoglobin: Ligand Dynamics in the First 10ps. Chem. Phys. 1991, 158, 221-248.

(15) Petrich, J. W.; Lambry, J. C.; Kuczera, K.; Karplus, M.; Poyart, C.; Martin, J. L. Ligand Binding and Protein Relaxation in Heme Proteins: A Room Temperature Analysis of NO Geminate Recombination. Biochemistry 1991, 30, 3975-3987.

(16) Schaad, O.; Zhou, H. X.; Szabo, A.; Eaton, W. A.; Henry, E. R. Simulation of the Kinetics of Ligand Binding to a Protein by Molecular Dynamics: Germinate Rebinding of Nitric Oxide to Myoglobin. Proc. Natl. Acad. Sci. U.S.A. 1993, 90, 9547-9551.

(17) Li, H.; Elber, R; Straub, J. E. Molecular Dynamics Simulation of NO Recombination to Myoglobin Mutants. J. Biol. Chem. 1993, 268, 17908-17916.

(18) Ma, J.; Huo, S.; Straub, J. E. Molecular Dynamics Simulation Study of the B-States of Solvated Carbon Monoxymyoglobin. J. Am. Chem. Soc. 1997, 119, 2541-2551.

(19) Sagnella, D. E.; Straub, J. E. Directed Energy "Funneling" Mechanism for Heme Cooling Following Ligand Photolysis or Direct Excitation in Solvated Carbonmonoxy Myoglobin. J. Phys. Chem. B 2001, 105, 7057-7063.

(20) Jensen, K. P.; Ryde, U. How O2 Binds to Heme. J. Biol. Chem. 2004, 279, 14561-14569.

(21) Dunietz, B. D.; Dreuw, A.; Head-Gordon, M. Initial Steps of the Photodissociation of the CO Ligated Heme Group. J. Phys. Chem. B 2003, 107, 5623-5629.

(22) Dreuw, A.; Dunietz, B. D.; Head-Gordon, M. Characterization of the Relevant Excited States in the Photodissociation of CO-Ligated Hemoglobin and Myoglobin. J. Am. Chem. Soc. 2002, 124, 1207012071.

(23) Harvey, J. N. DFT Computation of the Intrinsic Barrier to CO Germinate Recombination with Heme Compounds. J. Am. Chem. Soc. 2000, 122, 12401-12402.

(24) Frauenfelder, H.; Sligar, S. G.; Wolynes, P. G. The Energy Landscapes and Motions of Proteins. Science 1991, 254, 1598-1603.

(25) Gibson, Q. H.; Edelstein, J. S. Oxygen Binding and Subunit Interaction of Hemoglobin in Relation to the Two State Model. J. Biol. Chem. 1987, 262, 516-519.
(26) Olson, J. S.; Rohlfs, R. J.; Gibson, Q. H. Ligand Recombination to the $\alpha$ and $\beta$ Subunits of Human Hemoglobin. J. Biol. Chem. 1987, $262,12930-12938$.

(27) Gibson, Q. H.; Regan, R.; Elber, R.; Olson, J. S.; Carver, T. E. Distal Pocket Residues Affect Picosecond Ligand Recombination in Myoglobin. J. Biol. Chem. 1992, 267, 22022-22034.

(28) Carlson, M. L.; Regan, R.; Elber, R.; Li, H.; Phillips, G. N.; Olson, J. S.; Gibson, Q. H. NO Recombination to Double Mutants of Myoglobin: Role of Ligand Diffusion in a Fluctuating Heme Pocket. Biochemistry 1994, 33, 10597-10606.

(29) Jongeward, K. A.; Magde, D.; Taube, D. J.; Traylor, T. G. Picosecond Kinetics of Cytochromes b5 and C*. J. Biol. Chem. 1988, 263, 6027-6030.

(30) Agmon, N.; Sastry, G. M. Temperature-Dependent Effecitve Potential Explains CO Rebinding to Myoglobin. Chem. Phys. 1996, 212, 207-219.

(31) Agmon, N.; Doster, W.; Post, F. The Transition from Inhomogeneous to Homogeneous Kinetics in $\mathrm{CO}$ Binding to Myoglobin. Biophys. J. 1994, 66, 1612-1622.

(32) Austin, R.; Beeson, K.; Eisenstein, L.; Frauenfelder, H. Dynamics of Ligand Binding to Myoglobin. Biochemistry 1975, 14, 5355-5373.

(33) Nienhaus, G. U.; Mourant, J. R.; FRauenfelder, H. Spectroscopic Evidence for Conformational Relaxation in Myoglobin. Proc. Natl. Acad. Sci. U.S.A. 1992, 89, 2902-2906.

(34) Ionascu, D.; Gruia, F.; Ye, X.; Yu, A.; Rosca, F.; Beck, C.; Demidov, A.; Olson, J. S.; Champion, P. M. Temperature-Dependent Studies of NO Recombination to Heme and Heme Proteins. J. Am. Chem. Soc. 2005, 127, 16921-16934.

(35) Ye, X.; Ionascu, D.; Gruia, F.; Yu, A.; Benabbas, A.; Champion, P. M. Temperature-Dependent Heme Kinetics with Nonexponential Binding and Barrier Relaxation in the Absence of Protein Conformational Substates. Proc. Natl. Acad. Sci. U.S.A. 2007, 104, 14682-14687.

(36) Srajer, V.; Champion, P. M. Investigations of Optical Line Shapes and Kinetic Hole Burning in Myoglobin. Biochemistry 1991, 30, 7390-7402.

(37) Srajer, V.; Schomacker, K.; Champion, P. Spectral Broadening in Biomolecules. Phys. Rev. Lett. 1986, 57, 1267-1270.

(38) Zhang, P.; Malolepsza, E.; Straub, J. E. Dynamics of Methionine Ligand Rebinding in Cytochrome c. J. Phys. Chem. B 2012, 116, 69806990.

(39) Wang, W.; Ye, X.; Demidov, A. A.; Rosca, F.; Sjodin, T.; Cao, W.; Sheeran, M.; Champion, P. M. Femtosecond Multicolor PumpProbe Spectroscopy of Ferrous Cytochrome c. J. Phys. Chem. B 2000, 104, 10789-10801.

(40) Negrerie, M.; Cianetti, S.; Vos, M. H.; Martin, J. L.; Kruglik, S. G. Ultrafast Heme Dynamics in Ferrous Versus Ferric Cytochrome c Studied by Time-Resolved Resonance Raman and Transient Absorption Spectroscopy. J. Phys. Chem. B 2006, 110, 12766-12781.

(41) Vos, M. H.; Battistoni, A.; Lechauve, C.; Marden, M. C.; Kiger, L.; Desbois, A.; Pilet, E.; de Rosny, E.; Liebl, U. Ultrafast Heme Residue Bond Formation in Six-Coordinate Heme Proteins: Implications for Functional Ligand Exchange. Biochemistry 2008, 47, $5718-5723$.

(42) Zang, C.; Stevens, J. A.; Link, J. J.; Guo, L.; Wang, L.; Zhong, D. Ultrafast Proteinquake Dynamics in Cytochrome c. J. Am. Chem. Soc. 2009, 131, 2846-2852.

(43) Jorgensen, W.; Chandrasekhar, J.; Madura, J.; Impey, R.; Klein, M. Comparison of Simple Potential Functions for Simulating Liquid Water. J. Chem. Phys. 1983, 79, 926-935.

(44) Verlet, L. Computer Experiments on Classical Fluids. I. Thermodynamical Properties of Lennard-Jones Molecules. Phys. Rev. 1967, 1, 98-103.

(45) MacKerell, A. D.; Bashford, D.; Bellott, M.; Dunbrack, R. L.; Evanseck, J. D.; Field, M. J.; Fischer, S.; Gao, J.; Guo, H.; Ha, S.; et al. All-Atom Empirical Potential for Molecular Modeling and Dynamics Studies of Proteins. J. Phys. Chem. B 1998, 102, 3586-3616.

(46) Ryckaert, J.-P.; Ciccotti, G.; Berendsen, H. J. Numerical Integration of the Cartesian Equations of Motion of a System with 
Constraints: Molecular Dynamics of N-Alkanes. J. Comput. Phys. 1977, 23, 327-341.

(47) Amadei, A.; Linssen, A.; Berendsen, H. Essential Dyanmics of Proteins. Proteins 1993, 17, 412-425.

(48) Fischer, S.; Karplus, M. Conjugate Peak Refinement: an Algorithm for Finding Reaction Paths and Accurate Transition States in Systems with Many Degrees of Freedom. Chem. Phys. Lett. 1992, 194, 252-261.

(49) Frauenfelder, H.; Wolynes, P. G. Rate Theories and Puzzles of Hemeprotein Kinetics. Science 1985, 229, 337-345.

(50) Straub, J.; Berne, B. A Statistical Theory for the Effect of Nonadiabatic Transition on Activated Processes. J. Chem. Phys. 1987, 87, 6111-6116.

(51) Berne, B.; Borkovec, M.; Straub, J. Classical and Modern Methods in Reaction Rate Theory. J. Phys. Chem. 1988, 92, 37113725 .

(52) Biroli, G.; Garrahan, J. P. Perspective: The Glass Transition. J. Chem. Phys. 2013, 138, 12A301.

(53) Chandler, D.; Garrahan, J. Dynamics on the Way to Forming Glass: Bubbles in Space-Time. Annu. Rev. Phys. Chem. 2010, 61, 191217.

(54) Agmon, N.; Rabinovich, S. Diffusive Dynamics on Potential Energy Surfaces: Nonequilibrium CO Binding to Heme Proteins. J. Chem. Phys. 1992, 97, 7270-7286.

(55) Elber, R.; Karplus, M. Multiple Conformational States of Proteins: A Molecular Dynamics Analysis of Myoglobin. Science 1987, 235, 318-321.

(56) Friedman, M.; Rousseau, D. L.; Ondrias, M. R.; Stepnoski, R. A. Transient Raman Study of Hemoglobin:Structural Dependence of the Iron-Histidine Linkage. Science 1982, 218, 1244-1246.

(57) Gerstman, B. S. CO Binding to Hemoglobin: Spin-Tunneling Temperature, $\mathrm{Fe}$ Electronic States, and Electronic Effects on Nonadiabatic Dynamics. J. Chem. Phys. 1988, 88, 6228-6232.

(58) Sabelko, J.; Ervin, J.; Gruebele, M. Observation of Strange Kinetics in Protein Folding. Proc. Nat. Acad. Sci. USA 1999, 96, 60316036. 